\title{
Characteristics and Outcomes of Coronavirus Disease 2019 (COVID-19) Infection among Children Admitted in Al-Imam Abdulrahman Al Faisal Hospital in Riyadh, Saudi Arabia
}

\author{
Abdullah H. Alhamoud ${ }^{1,2^{*}}$, Hareth A. Aldosaimani², Abdullah A. Alyahya ${ }^{2}$, Ahmad K. Almadani ${ }^{2}$, \\ Nabil H. Madkhali ${ }^{3}$, Laila M. Alali ${ }^{4}$, Ibrahim A. Alsahabi ${ }^{2}$ \\ ${ }^{1}$ Department of Laboratory, Al-Imam Abdulrahman Al-Faisal Hospital, First Health Cluster, Ministry of Health, \\ Riyadh, Saudi Arabia \\ ${ }^{2}$ Department of Pediatrics, Al-Imam Abdulrahman Al-Faisal Hospital, First Health Cluster Ministry of Health, \\ Riyadh, Saudi Arabia \\ ${ }^{3}$ Department of Health Management, Al-Imam Abdulrahman Al-Faisal Hospital, First Health Cluster Ministry of Health, \\ Riyadh, Saudi Arabia \\ ${ }^{4}$ Collage of Medicine, Jazan Univerisity, Jazan, Saudi Arabia \\ Email: *dr.alhamoud1990@gmail.com
}

How to cite this paper: Alhamoud, A.H., Aldosaimani, H.A., Alyahya, A.A., Almadani, A.K., Madkhali, N.H., Alali, L.M. and Alsahabi, I.A. (2021) Characteristics and Outcomes of Coronavirus Disease 2019 (COVID-19) Infection among Children Admitted in Al-Imam Abdulrahman Al Faisal Hospital in Riyadh, Saudi Arabia. Open Journal of Pediatrics, 11, 170-178. https://doi.org/10.4236/ojped.2021.112016

Received: February 9, 2021

Accepted: May 11, 2021

Published: May 14, 2021

Copyright () 2021 by author(s) and Scientific Research Publishing Inc. This work is licensed under the Creative Commons Attribution International License (CC BY 4.0).

http://creativecommons.org/licenses/by/4.0/

(c) (i) Open Access

\begin{abstract}
Background: At the beginning of the COVID-19 pandemic, some reports demonstrated that children are at lower risk of infection. Though, with the growing transmission of the virus, it has been illustrated that it can infect children. Nevertheless, the features and outcomes of COVID-19 infection in children are still unclear. Objective: This study aims to describe the clinical characters and outcomes of children infected with COVID-19 and admitted to a hospital in Saudi Arabia. Design and Setting: This is a retrospective study that was carried out in Imam Abdulrahman Al Faisal Hospital in Riyadh, Saudi Arabia, by collecting data from patients' records of children who were admitted to the hospital from March to July 2020. The collected data included information on the age and gender of the children, as well as their clinical course during hospitalization. Results: Thirty-three patients were included; $39.4 \%$ of the children aged less than one year, $51.5 \%$ were males. Asthma occurred in $15.2 \%$ of patients. Asthma ( $\mathrm{p}$-value $=0.035$ ), and chronic lung disease ( $\mathrm{p}$-value $=0.046)$ were the most significantly occurring comorbidities. Also, $72.7 \%$ of the children were admitted to the ward, while only $3 \%$ required a neonatal intensive care unit (NICU) and children care unit (PICU). The most prevalent manifestation was fever in $45.5 \%$. Fever ( $p$-value $=0.024)$, cough and headache among children more than six years ( $\mathrm{p}$-value $<0.001$ ),
\end{abstract}


and gastrointestinal symptoms $(\mathrm{p}$-value $=0.038)$ were the most significant symptoms. Temperature $>38$ ( $\mathrm{p}$-value $=0.043$ ), oxygen saturation $<94 \%$ $(\mathrm{p}$-value $=0.021)$, low systolic blood pressure and high heart rate ( $\mathrm{p}$-value $<$ 0.001) were the most common vital signs abnormalities. Of the whole patients, 3\% showed abnormal radiographic investigation. The mean length of hospital stay was $8.9 \pm 4.2$ days, no in-hospital COVID-19 related mortality was reported. Conclusion: The prognosis of children with COVID-19 who are hospitalized was relatively good, with no in-hospital COVID-19 related mortality identified. A larger study is needed to confirm the outcomes of the present study.

\section{Keywords}

COVID-19, Clinical Outcomes, Children, Characters, Mortality, Saudi Arabia

\section{Introduction}

Since the early identification of COVID-19 infection in an adult patient in Wuhan city, China, the most serious concern was with the acute respiratory distress syndrome caused by the infection [1], severe respiratory manifestations were the predominant reason for hospitalization and the need for more invasive interventions, up to invasive mechanical ventilation [2]. Consequently, most of the research studies focused on examining and explaining these respiratory features in adults without any thought of infection in children [3].

Although early reports from different countries showed a low risk of COVID-19 infection among children, with the increasing experience with the pandemic, it has been revealed that it can also affect the children population, though most of the detected cases had mild symptoms with good prognosis [4] [5]. The clinical features in children were almost similar to adults in terms of the presence of respiratory symptoms and gastrointestinal symptoms [6].

There are scarce data on the COVID-19 infection features in children. It has been demonstrated that $95 \%$ of children with a positive polymerase chain reaction (PCR) for COVID-19 were asymptomatic [7]. However, only $5 \%$ of the symptomatic children showed significant symptoms of respiratory distress such as dyspnea and hypoxemia. This percentage is considerably lower than that identified in adults [8]. Fortunately, less than $1 \%$ of infected children can progress to multiorgan failure and acute respiratory distress syndrome [9].

Recently, with the beginning of the second wave of the COVID-19 pandemic, the number of children with COVID-19 infections has been increasing [10]. Additionally, a growing number of intensive care admissions among children have been seen [11]. Despite these alarming figures, there is a scarcity of information about the presentation and treatment outcomes of children with severe infection who require hospital admissions [12].

Understanding the clinical features and course of hospitalization of children 
infected with COVID-19 is essential in order to design appropriate treatment strategies in this special population and improve patients' outcomes [13]. Accordingly, the present study aims to understand the clinical characters and outcomes of hospitalized children infected with COVID-19 in Saudi Arabia.

\section{Materials and Methods}

\subsection{Study Design}

This is a retrospective study that included children who were admitted to Al-Imam Abdurrahman Al Faisal, first health cluster, ministry of health, Riyadh, Saudi Arabia. The inclusion criteria were children who were admitted during the COVID-19 pandemic from March to July 2020. Patients who did not meet COVID-19 diagnostic criteria were excluded.

\subsection{Data Collection}

A predesigned excel sheet was used for the purpose of the retrospective collection of patients' information. The collected data included demographic information about the children, their vital signs, clinical symptoms at presentation, history of travel, or relatives working in healthcare facilities. Also, the outcome of hospitalization and treatment were recorded.

\subsection{Statistical Analyses}

Statistical analysis was performed using SPSS version 26. Descriptive analysis was carried out using counts and percentages for categorical variables, while numerical variables used means and standard deviations. Identifying the most significant characters and features correlated to COVID-19 infection was done through one sample chi-square testing at a level of significance $\mathrm{p}$-value $<0.05$.

\subsection{Ethical Considerations}

Ethical approval from the study site was obtained before the data collection. The identity of the patients was masked throughout the study.

\section{Results}

Thirty-three patients were eligible for inclusion. Descriptive analysis is described below.

\subsection{Demographic Characters of Children}

Out of 33 patients, the age was categorized into four groups, where $39.4 \%$ of the children aged less than one year, while $36.4 \%$ aged above six years old. As for the gender, $51.5 \%$ were males, as described in Table 1.

\subsection{Comorbidities}

The comorbidities of the children were analyzed. Asthma was the most common 
comorbidity occurring in $15.2 \%$ of the patients, followed by cancer and immunodeficiency and chronic lung disease, with a prevalence of $6.1 \%$ for each. One sample chi-square test was used to identify the most significant comorbidities occurring with COVID-19 infection among children. It has been demonstrated that asthma $(\mathrm{p}$-value $=0.035)$ and chronic lung disease $(\mathrm{p}$-value $=0.046)$ were the most significantly occurring comorbidities, as described in Table 2.

\subsection{Clinical Outcome and Admission Characters}

Upon assessment, patients were admitted to different departments. $72.7 \%$ of the children were admitted to the ward, while only $3 \%$ required a neonatal intensive care unit (NICU) and pediatric care unit (PICU), as shown in Figure 1.

Table 1. Demographic information of the children.

\begin{tabular}{cccc}
\hline & & Count & Percent \\
\hline less than one year & 13 & $39.4 \%$ \\
Age Category & 1 to 5 & 8 & $24.2 \%$ \\
& 6 to 10 & 6 & $18.2 \%$ \\
More than 10 & 6 & $18.2 \%$ \\
\hline \multirow{2}{*}{ Gender } & Female & 16 & $48.5 \%$ \\
& Male & 17 & $51.5 \%$
\end{tabular}

Table 2. Comorbidities.

\begin{tabular}{cccc}
\hline Comorbidities & Count & Percent & P-value \\
\hline Asthma & 5 & $15.2 \%$ & 0.035 \\
Cancer/Immunodeficiency & 2 & $6.1 \%$ & 0.987 \\
Chronic lung disease & 2 & $6.1 \%$ & 0.046 \\
Cardiac disease & 1 & $3.0 \%$ & 0.721 \\
Hypertension & 1 & $3.0 \%$ & 0.684 \\
Diabetes mellitus & 1 & $3.0 \%$ & 0.654
\end{tabular}

${ }^{*} \mathrm{p}$-value at the level of significance $<0.05$.

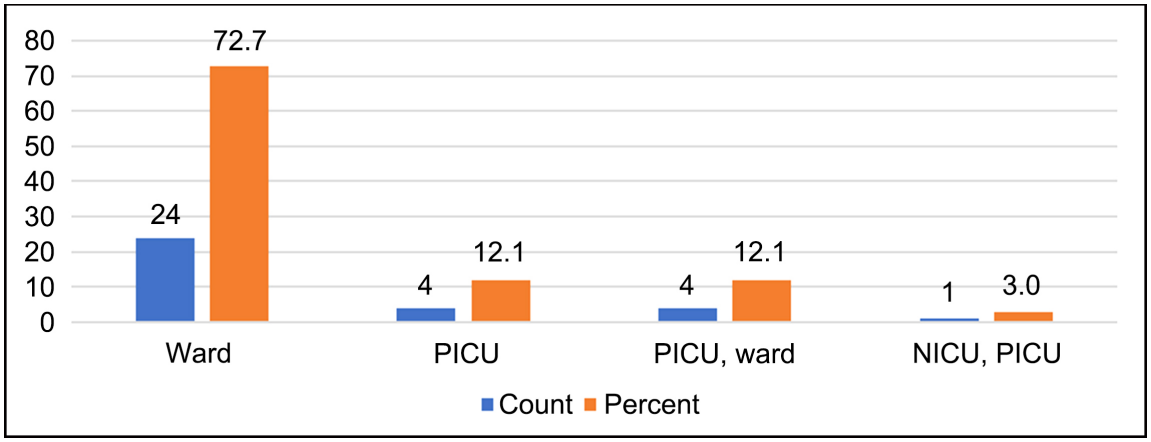

Figure 1. Admission of children to different departments. 


\subsection{Laboratory Results}

Laboratory results of the included patients were also evaluated. It has been shown that a significant lymphocytosis and high level of platelets count among eight patients.

\subsection{Symptoms and Clinical Features}

As for the symptoms of patients, the most common symptom was fever in $45.5 \%$, followed by cough in $39.4 \%$, while the least common symptom was myalgia, occurring in $3 \%$ of the full cohort. In order to identify the most significant symptoms correlated to COVID-19 infection in children, the one-sample chi-square test was used at the level of significance $p$-value $<0.05$.

It has been shown that fever $(\mathrm{p}$-value $=0.024)$, cough and headache (headache demonstrated in patient age from six and older) ( $\mathrm{p}$-value $<0.001$ ), and gastrointestinal symptoms $(\mathrm{p}$-value $=0.038$ ) were the most significant symptoms among COVID-19 patients, as described in Table 3.

Table 3. COVID-19 symptoms among patients.

\begin{tabular}{cccc}
\hline & Count & Percent & P-value \\
\hline Fever & 15 & $45.5 \%$ & $0.024^{*}$ \\
Cough & 13 & $39.4 \%$ & $<0.001^{\star}$ \\
Headache & 10 out of 12 & $80.3 \%$ & $<0.001^{*}$ \\
Gastrointestinal symptoms & 9 & $27.3 \%$ & $0.038^{*}$ \\
Runny nose & 3 & $9.1 \%$ & 0.084 \\
Sore throat & 2 & $6.1 \%$ & 0.095 \\
Myalgia & 1 & $3.0 \%$ & 0.953 \\
\hline
\end{tabular}

${ }^{*} \mathrm{p}$-value at the level of significance $<0.05$

\subsection{Vital Signs}

Vital signs and oxygenation are varient among children depened on age group (Table 4). These variables were reported and analyzed. It has been shown that temperature $>38^{\circ} \mathrm{C}$ was the most common feature in $42.4 \%$ of patients, followed by oxygen saturation less than $94 \%$ in $18.2 \%$. using one-sample chi-square test, it has been shown that temperature $>38(\mathrm{p}$-value $=0.043)$, oxy- gen saturation $<$ $94(\mathrm{p}$-value $=0.021)$, low systolic blood pressure, and high heart rate $(\mathrm{p}$-value < 0.001 ) were the most common vital signs accompanying COVID-19 infection in hospitalized children, as described in Table 5.

\subsection{Radiographic Manifestations}

Results from radiographic manifestations were reported. $87.9 \%$ of the children had a normal examination, while only $3 \%$ had pulmonary edema not related to COVID-19 infection, and another 3\% showed abnormal radiographic investigation, as shown in Figure 2. 
Table 4. Vital signs normal ranges.

\begin{tabular}{|c|c|c|c|c|c|c|}
\hline Age Vital signs & $1-12$ months & 1 - 2 years & 3 - 5 years & $6-11$ years & $12-15$ years & \\
\hline Heart rare & $100-190$ & $98-140$ & $80-120$ & $75-118$ & $60-100$ & \\
\hline Respiratory rate & $22-37$ & $20-28$ & $20-28$ & $18-25$ & $12-20$ & \\
\hline Age Vital signs & $1-12$ months & 1 - 2 years & 3 - 5 years & 6 - 9 years & $10-11$ years & $12-15$ years \\
\hline Systolic blood pressure & $72-104$ & $86-106$ & $89-112$ & $97-115$ & $102-120$ & $110-131$ \\
\hline
\end{tabular}

Table 5. COVID-19 vital signs among patients.

\begin{tabular}{cccc}
\hline & Count & Percent & P-value \\
\hline Temperature $>38^{\circ} \mathrm{C}$ & 14 & $42.4 \%$ & 0.043 \\
Oxygen saturation $<94 \%$ & 6 & $18.2 \%$ & 0.021 \\
Low abnormal Systolic blood pressure & 4 & $12.1 \%$ & $<0.001$ \\
High abnormal Heart rate & 4 & $12.1 \%$ & $<0.001$ \\
Abnormal respiratory rate & 2 & $6.1 \%$ & 0.364 \\
\hline
\end{tabular}

${ }^{*} \mathrm{p}$-value at level of significance $<0.05$; ${ }^{*}$ vital signs based on age in appendix.

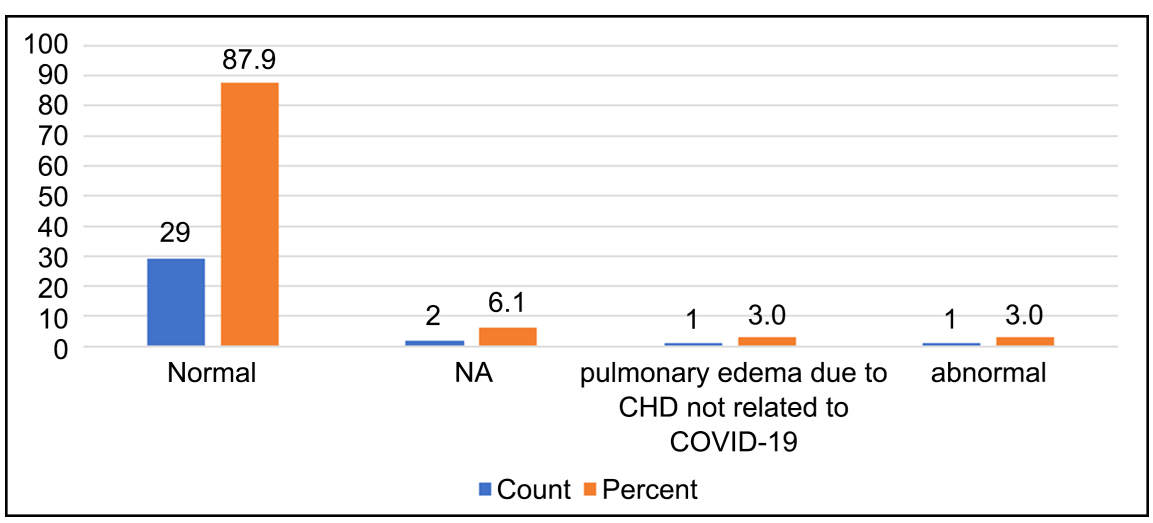

Figure 2. Radiographic manifestation.

\subsection{Other Features and Outcomes}

It has been revealed that none of the patients had in-hospital COVID-19 related mortality. Also, no patients had a history of recent travel abroad, and none of the patients' relatives worked in a healthcare facility. The average length of hospital stay was $8.9 \pm 4.2$ days, with a minimum of two days and a maximum of 18 days.

\section{Discussion}

COVID-19 infections in the children population are underestimated. This could be referred to the asymptomatic nature of the disease in most of the children [14]. However, children who have symptoms and require hospitalization usually present with deteriorating respiratory conditions [15]. These children may need advanced respiratory support strategy and sometimes intensive care unit admission. Though the full clinical features of COVID-19 infection are still not clearly 
understood [16].

The present study aimed to understand the clinical characters and clinical outcomes of COVID-19 infection in a small cohort. It has been demonstrated that the prognosis of the children hospitalized with COVID-19 is generally good, with no in-hospital mortalities occurring during the study duration. Also, the average hospital stay was approximately one week. Additionally, the infection did not appear to be related to a history of travel or work of relatives in a healthcare setting.

Concerning the clinical features of the infection, it has been shown that hospitalization with COVID-19 infection among children was significantly correlated to history of asthma $(\mathrm{p}$-value $=0.035)$ and chronic lung disease $(\mathrm{p}$-value $=$ $0.046)$, cough and headache ( $\mathrm{p}$-value $<0.001$ ), and gastrointestinal symptoms $(\mathrm{p}$-value $=0.038)$, temperature $>38^{\circ} \mathrm{C}(\mathrm{p}$-value $=0.043)$, oxygen saturation $<$ $94 \%(\mathrm{p}$-value $=0.021)$, low systolic blood pressure and high heart rate $(\mathrm{p}$-value $<$ $0.001)$.

Some data do exist on COVID-19 infections in children. A systematic review by Ludvigsson et al. [17] examined the prognosis and clinical manifestations of COVID-19 among children. Ludvigsson et al. [17] demonstrated that symptoms of the infection in children are mild and showed a better prognosis compared to adults. Additionally, Ludvigsson et al. [17] described mortality among children as "extremely rare", as well as reporting cases with COVID-19 in children less than two years old.

Similar to Ludvigsson et al. [17], the present study demonstrated that more than a third of the included cohort was less than one year old. Furthermore, none of the children died from COVID-19 during hospitalization, and all the children had mild severity of symptoms, where the symptoms were similar to symptoms reported in adult patients.

Also, a study by Li et al. [18] evaluated the radiographic findings of chest computed tomography in the children population who were admitted to a Chinese tertiary hospital. Only five children were eligible for inclusion. Li et al. [18] showed that almost half of the children had an abnormal finding on the computed tomography. This abnormality was in the form of ground glass appearance, which changed to normal after medical management [18].

The present study also included the radiographic measurements of the included children. Unlike Li et al. [18], 87.9\% of the children had a normal radiographic scan, where only one patient had a non-COVID-19 related abnormality, and only one patient had a COVID-19 related abnormality. It should be noted that the sample included in the present study is quite larger than that included in Li et al. [18].

Another study by Li et al. [19] examined the clinical features of COVID-19 infection in twenty-two children with COVID-19 infection in China. Li et al. [19] showed that the most significant symptoms occurring were fever and cough. However, the symptoms were generally mild, with no reported COVID-19 re- 
lated mortality. These findings are in compliance with the findings of the present study and the findings from Ludvigsson et al. [17].

The present study had some limitations; the sample size was relatively small, which could be attributed to the few numbers of children presenting to the hospital with COVID-19 symptoms. Hence, future studies should consider including a larger sample size. Also, the present study was performed in one hospital; this could limit the external validity of the study outcomes. Therefore, multicenter studies are highly recommended.

\section{Conclusion}

Children patients with COVID-19 infections usually have mild symptoms and normal radiographic examination. Fever is the most common clinical manifestation, with a temperature above $38^{\circ} \mathrm{C}$ is the most common vital sign abnormality seen. Also, the prognosis of these patients appeared to be good. However, larger and prospective studies are needed to confirm these findings. Through this study, the research team would recommend further testing of COVID-19 in the children population, which is underestimated.

\section{Conflicts of Interest}

The authors declare no conflicts of interest regarding the publication of this paper.

\section{References}

[1] Phelps, C. and Sperry, L.L. (2020) Children and the COVID-19 Pandemic. Psychological Trauma: Theory, Research, Practice, and Policy, 12, S73-S75. https://doi.org/10.1037/tra0000861

[2] She, J., Liu, L. and Liu, W. (2020) COVID-19 Epidemic: Disease Characteristics in Children. Journal of Medical Virology, 92, 747-754. https://doi.org/10.1002/jmv.25807

[3] Lee, P.I., Hu, Y.L., Chen, P.Y., Huang, Y.C. and Hsueh, P.R. (2020) Are Children Less Susceptible to COVID-19? Journal of Microbiology, Immunology, and Infection, 53, 371-372. https://doi.org/10.1016/j.jmii.2020.02.011

[4] Liu, W., Zhang, Q., Chen, J., Xiang, R., Song, H., Shu, S., Chen, L., Liang, L., Zhou, J., You, L. and Wu, P. (2020) Detection of COVID-19 in Children in Early January 2020 in Wuhan, China. New England Journal of Medicine, 382, 1370-1371. https://doi.org/10.1056/NEJMc2003717

[5] Riphagen, S., Gomez, X., Gonzalez-Martinez, C., Wilkinson, N. and Theocharis, P. (2020) Hyperinflammatory Shock in Children during COVID-19 Pandemic. The Lancet, 395, 1607-1608. https://doi.org/10.1016/S0140-6736(20)31094-1

[6] Masonbrink, A.R. and Hurley, E. (2020) Advocating for Children during the COVID-19 School Closures. Pediatrics, 146, e20201440. https://doi.org/10.1542/peds.2020-1440

[7] Dong, Y., Mo, X., Hu, Y., Qi, X., Jiang, F., Jiang, Z. and Tong, S. (2020) Epidemiology of COVID-19 among Children in China. Pediatrics, 145, e20200702. https://doi.org/10.1542/peds.2020-0702

[8] Cruz, A.T. and Zeichner, S.L. (2020) COVID-19 in Children: Initial Characteriza- 
tion of the Pediatric Disease. Pediatrics, 145, e20200834.

https://doi.org/10.1542/peds.2020-0834

[9] Lee, B. and Raszka, W.V. (2020) COVID-19 Transmission and Children: The Child Is Not to Blame. Pediatrics, 146, e2020004879.

https://doi.org/10.1542/peds.2020-004879

[10] Ranabothu, S., Onteddu, S., Nalleballe, K., Dandu, V., Veerapaneni, K. and Veerapandiyan, A. (2020) Spectrum of COVID-19 in Children. Acta Paediatrica, 109, 1899-1900. https://doi.org/10.1111/apa.15412

[11] Kelvin, A.A. and Halperin, S. (2020) COVID-19 in Children: The Link in the Transmission Chain. The Lancet Infectious Diseases, 20, 633-634.

https://doi.org/10.1016/S1473-3099(20)30236-X

[12] Brodin, P. (2020) Why Is COVID-19 So Mild in Children? Acta Paediatrica, 109, 1082-1083. https://doi.org/10.1111/apa.15271

[13] Götzinger, F., Santiago-García, B., Noguera-Julián, A., Lanaspa, M., Lancella, L., Carducci, F.I., Gabrovska, N., Velizarova, S., Prunk, P., Osterman, V. and Krivec, U. (2020) COVID-19 in Children and Adolescents in Europe: A Multinational, Multicentre Cohort Study. The Lancet Child \& Adolescent Health, 4, 653-661. https://doi.org/10.1016/S2352-4642(20)30177-2

[14] Ludvigsson, J.F. (2020) Children Are Unlikely to Be the Main Drivers of the COVID-19 Pandemic-A Systematic Review. Acta Paediatrica, 109, 1525-1530.

https://doi.org/10.1111/apa.15371

[15] Kim, L., Whitaker, M., O’Halloran, A., Kambhampati, A., Chai, S.J., Reingold, A., Armistead, I., Kawasaki, B., Meek, J., Yousey-Hindes, K. and Anderson, E.J. (2020) Hospitalization Rates and Characteristics of Children Aged $<18$ Years Hospitalized with Laboratory-Confirmed COVID-19-COVID-NET, 14 States, March 1-July 25, 2020. Morbidity and Mortality Weekly Report, 69, 1081-1088. https://doi.org/10.15585/mmwr.mm6932e3

[16] Parri, N., Lenge, M. and Buonsenso, D. (2020) Children with COVID-19 in Pediatric Emergency Departments in Italy. The New England Journal of Medicine, 383, 187-190. https://doi.org/10.1056/NEJMc2007617

[17] Ludvigsson, J.F. (2020) Systematic Review of COVID-19 in Children Shows Milder Cases and a Better Prognosis than Adults. Acta Paediatrica, 109, 1088-1095. https://doi.org/10.1111/apa.15270

[18] Li, W., Cui, H., Li, K., Fang, Y. and Li, S. (2020) Chest Computed Tomography in Children with COVID-19 Respiratory Infection. Pediatric Radiology, 50, 769-799. https://doi.org/10.1007/s00247-020-04656-7

[19] Li, B., Shen, J., Li, L. and Yu, C. (2020) Radiographic and Clinical Features of Children with Coronavirus Disease (COVID-19) Pneumonia. Indian Pediatrics, 57, 423-426. https://doi.org/10.1007/s13312-020-1816-8 